Proc. Indian Acad. Sci. (Math. Sci.) Vol. 115, No. 1, February 2003, pp. 93-102.

Printed in India

\title{
On two functionals connected to the Laplacian in a class of doubly connected domains in space-forms
}

\author{
M H C ANISA and A R AITHAL \\ Department of Mathematics, University of Mumbai, Mumbai 400 098, India \\ E-mail: anisa@sankhya.mu.ac.in; aithal@math.mu.ac.in
}

MS received 7 September 2004; revised 15 December 2004

\begin{abstract}
Let $B_{1}$ be a ball of radius $r_{1}$ in $S^{n}\left(\mathbb{H}^{n}\right)$, and let $B_{0}$ be a smaller ball of radius $r_{0}$ such that $\overline{B_{0}} \subset B_{1}$. For $S^{n}$ we consider $r_{1}<\pi$. Let $u$ be a solution of the problem $-\Delta u=1$ in $\Omega:=B_{1} \backslash \overline{B_{0}}$ vanishing on the boundary. It is shown that the associated functional $J(\Omega)$ is minimal if and only if the balls are concentric. It is also shown that the first Dirichlet eigenvalue of the Laplacian on $\Omega$ is maximal if and only if the balls are concentric.
\end{abstract}

Keywords. Eigenvalue problem; Laplacian; maximum principles.

\section{Introduction}

Let $(M, g)$ be a Riemannian manifold and let $D$ denote the Levi-Civita connection of $(M, g)$. For a smooth vector field $X$ on $M$ the divergence $\operatorname{div}(X)$ is defined as $\operatorname{trace}(D X)$. For a smooth function $f: M \longrightarrow \mathbb{R}$, the gradient $\nabla f$ is defined by $g(\nabla f(p), v)=\mathrm{d} f(p)(v)$ $\left(p \in M, v \in T_{p} M\right)$ and the Laplace-Beltrami operator $\Delta$ is defined by $\Delta f=\operatorname{div}(\nabla f)$. Further, $\nabla^{2} f$ denotes the Hessian of $f$. Throughout this paper, $\omega$ and $\mathrm{d} V$ denote the volume element of $(M, g)$.

Let $\Omega \subset M$ be a domain such that $\bar{\Omega}$ is a smooth compact submanifold of $M$. The Sobolev space $H^{1}(\Omega)$ is defined as the closure of $\mathscr{C}^{\infty}(\bar{\Omega})$ (the space of real valued smooth functions on $\bar{\Omega}$ ) with respect to the Sobolev norm

$$
\|f\|_{H^{1}(\Omega)}=\left(\int_{\Omega}\left\{f^{2}+\|\nabla f\|^{2}\right\} \mathrm{d} V\right)^{1 / 2} \quad\left(f \in \mathscr{C}^{\infty}(\bar{\Omega})\right) .
$$

The closure of $\mathscr{C}_{0}^{\infty}(\Omega)$ (the space of real valued smooth functions on $\Omega$ having compact support in $\Omega$ ) in $H^{1}(\Omega)$ is denoted by $H_{0}^{1}(\Omega)$. The Sobolev space $H^{2}(\Omega)$ is defined as the closure of $\mathscr{C}^{\infty}(\bar{\Omega})$ with respect to the Sobolev norm

$$
\|f\|_{H^{2}(\Omega)}=\left(\int_{\Omega}\left\{f^{2}+\|\nabla f\|^{2}+\left\|\nabla^{2} f\right\|^{2}\right\} \mathrm{d} V\right)^{1 / 2} \quad\left(f \in \mathscr{C}^{\infty}(\bar{\Omega})\right) .
$$

These spaces are Hilbert spaces with the corresponding norms.

Consider the Dirichlet boundary value problem on $\Omega$ :

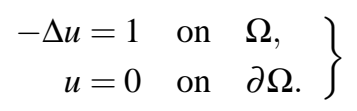


Let $u \in H_{0}^{1}(\Omega)$ be the unique weak solution of problem (1.1). By Theorem 4.8, p. 105 of [1], $u \in \mathscr{C}^{\infty}(\bar{\Omega})$.

Consider the following eigenvalue problem on $\Omega$ :

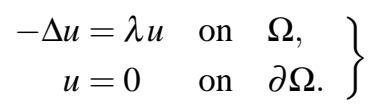

The eigenvalues of the positive Laplace-Beltrami operator $-\Delta=-\operatorname{div}(\nabla f)$ are strictly positive. The eigenfunctions corresponding to the first eigenvalue $\lambda_{1}$ are proportional to each other. They belong to $\mathscr{C}^{\infty}(\bar{\Omega})$ and they are either strictly positive or strictly negative on $\Omega$. Moreover,

$$
\lambda_{1}=\inf \left\{\|\nabla \phi\|_{L^{2}(\Omega)}^{2} \mid \phi \in H_{0}^{1}(\Omega),\|\phi\|_{L^{2}(\Omega)}^{2}=1\right\}
$$

(cf. [1], Theorem 4.4, p. 102). Let $y:=y(\Omega) \in \mathscr{C}^{\infty}(\bar{\Omega})$ be the unique solution of problem (1.1). Let $y_{1}:=y_{1}(\Omega)$ be the unique solution of problem (1.2), corresponding to the first eigenvalue $\lambda_{1}:=\lambda_{1}(\Omega)$, characterized by

$$
y_{1}>0 \quad \text { on } \Omega \text { and } \int_{\Omega} y_{1}^{2} \mathrm{~d} V=1 .
$$

The aim of this paper is to prove the main results of [3] for simply connected spherical and hyperbolic space-forms.

Consider the unit sphere $S^{n}=\left\{\left(x_{1}, x_{2}, \ldots, x_{n+1}\right) \in \mathbb{R}^{n+1} \mid \sum_{i=1}^{n+1} x_{i}^{2}=1\right\}$ with induced Riemannian metric $\langle$,$\rangle from the Euclidean space \mathbb{R}^{n+1}$. Also consider the hyperbolic space $\mathbb{H}^{n}=\left\{\left(x_{1}, x_{2}, \ldots, x_{n+1}\right) \in \mathbb{R}^{n+1} \mid \sum_{i=1}^{n} x_{i}^{2}-x_{n+1}^{2}=-1\right.$ and $\left.x_{n+1}>0\right\}$ with the Riemannian metric induced from the quadratic form $(x, y):=\sum_{i=1}^{n} x_{i} y_{i}-x_{n+1} y_{n+1}$, where $x=\left(x_{1}, x_{2}, \ldots, x_{n+1}\right)$ and $y=\left(y_{1}, y_{2}, \ldots, y_{n+1}\right)$.

Fix $0<r_{0}<r_{1}$. We choose $r_{1}<\pi$ for the case of $S^{n}$. Let $B_{1}$ be any ball of radius $r_{1}$ in $S^{n}\left(\mathbb{H}^{n}\right)$ and $B_{0}$ be any ball of radius $r_{0}$ such that $\overline{B_{0}} \subset B_{1}$. Consider the family $\mathscr{F}=\left\{B_{1} \backslash \overline{B_{0}}\right\}$ of domains in $S^{n}\left(\mathbb{H}^{n}\right)$. We study the extrema of the following functionals:

$$
\begin{aligned}
J(\Omega) & =-\int_{\Omega}\left\{\|\nabla y(\Omega)\|^{2}-2 y(\Omega)\right\} \mathrm{d} V, \\
J_{1}(\Omega) & =-\int_{\Omega}\left\{\left\|\nabla y_{1}(\Omega)\right\|^{2}-2 \lambda_{1}(\Omega)\left[y_{1}(\Omega)\right]^{2}\right\} \mathrm{d} V
\end{aligned}
$$

on $\mathscr{F}$, associated to problems (1.1) and (1.2) respectively. Note here that the functionals $J$ and $J_{1}$ are nothing but negative of the energy functional $\int_{\Omega}\|\nabla y(\Omega)\|^{2} \mathrm{~d} V$ and the Dirichlet eigenvalue $\lambda_{1}$, respectively.

We state our main results: Put $\Omega_{0}=B\left(p, r_{1}\right) \backslash \overline{B\left(p, r_{0}\right)}$ for any fixed $p \in S^{n}\left(\mathbb{H}^{n}\right)$.

Theorem 1. The functional $J(\Omega)$ on $\mathscr{F}$ assumes minimum at $\Omega$ if and only if $\Omega=\Omega_{0}$, i.e., when the balls are concentric.

Theorem 2. The functional $J_{1}(\Omega)$ on $\mathscr{F}$ assumes maximum at $\Omega$ if and only if $\Omega=\Omega_{0}$, i.e., when the balls are concentric.

In $\S \S 2$ and 3, following [5], we develop the 'shape calculus' for Riemannian manifolds for the stationary problem (1.1) and the eigenvalue problem (1.2) respectively. In $\S 4$, we prove Theorems 1 and 2 for $S^{n}$, and make the necessary remarks to carry out the proofs of Theorems 1 and 2 for $\mathbb{H}^{n}$. 


\section{Shape calculus for the stationary problem}

Let $V$ be a smooth vector field on $M$ having compact support. Let $\Phi: \mathbb{R} \times M \longrightarrow M$ be the smooth flow for $V$. For each $t \in \mathbb{R}$, denote $\Phi(t, x)$ by $\Phi_{t}(x)(x \in M)$. Let $\Omega$ be an open subset of $M$ such that $\bar{\Omega}$ is a smooth compact submanifold of $M$. Put $\Omega_{t}:=\Phi_{t}(\Omega)(t \in \mathbb{R})$.

Let $\mathscr{D}$ be a domain in $M$ such that $\operatorname{supp} V \subset \mathscr{D}$. Fix $f \in \mathscr{C}^{\infty}(\mathscr{D})$. Consider the Dirichlet boundary value problem on $\Omega_{t}$ :

$$
\left.\begin{array}{rll}
\Delta u=f & \text { on } & \Omega_{t}, \\
u=0 & \text { on } & \partial \Omega_{t} .
\end{array}\right\}
$$

Let $y_{t} \in \mathscr{C}^{\infty}\left(\bar{\Omega}_{t}\right)$ be the unique solution of problem (2.1) (cf. [1], Theorem 4.8, p. 105). Throughout this section $y:=y(\Omega)$ denotes the unique solution of (2.1) for $t=0$.

Denote $\left.y_{t} \circ \Phi_{t}\right|_{\Omega}$ by $y^{t}(t \in \mathbb{R})$.

\section{PROPOSITION 2.1.}

The map $t \longmapsto y^{t}$ is a $\mathscr{C}^{1}$-curve in $H^{2}(\Omega) \cap H_{0}^{1}(\Omega)$ from a neighbourhood of 0 in $\mathbb{R}$.

Proof. By problem (2.1), for each $t \in \mathbb{R}, y_{t}$ satisfies the equation

$$
\int_{\Omega_{t}} g\left(\nabla y_{t}, \nabla \psi\right) \mathrm{d} V=-\int_{\Omega_{t}} f \psi \mathrm{d} V \quad \forall \psi \in \mathscr{C}_{0}^{\infty}\left(\Omega_{t}\right) .
$$

There exists smooth function $\gamma_{t}: M \longrightarrow(0, \infty)$ such that $\Phi_{t}^{*} \omega=\gamma_{t} \omega$ (here, $\omega:=\mathrm{d} V$, the volume element of $(M, g)$ ). Put $B_{t}:=\left(D \Phi_{t}\right)^{-1}, B_{t}^{*}=$ transpose of $B_{t}$ (i.e., $g\left(B_{t}(x) v, w\right)=$ $g\left(v, B_{t}^{*}(x) w\right) \quad \forall v \in T_{x} \Omega_{t}, w \in T_{x^{\prime}} \Omega$, where $\left.x^{\prime}:=\Phi_{t}^{-1}(x)\right)$ and $A_{t}:=\gamma_{t} B_{t} B_{t}^{*}$. By the change of variable $\Phi_{t}: \Omega \longrightarrow \Omega_{t}$, eq. (3) can be re-written as

$$
\int_{\Omega}-\operatorname{div}\left(A_{t} \nabla\left(y_{t} \circ \Phi_{t}\right)\right) \psi \circ \Phi_{t} \mathrm{~d} V=-\int_{\Omega} f \circ \Phi_{t} \psi \circ \Phi_{t} \gamma_{t} \mathrm{~d} V .
$$

Therefore, $y^{t}:=y_{t} \circ \Phi_{t}: \Omega \longrightarrow \mathbb{R}$ satisfies

$$
\left.\begin{array}{rlll}
-\operatorname{div}\left(A_{t} \nabla y^{t}\right)+f \circ \Phi_{t} \gamma_{t}=0 & & \text { on } & \Omega, \\
y^{t}=0 & \text { on } & \partial \Omega .
\end{array}\right\}
$$

Define $F: \mathbb{R} \times H^{2}(\Omega) \cap H_{0}^{1}(\Omega) \longrightarrow L^{2}(\Omega)$ by $F(t, u)=-\operatorname{div}\left(A_{t} \nabla u\right)+f \circ \phi_{t} \gamma_{t}$. Then $F$ is a $\mathscr{C}^{1}$-map. Further $\left.D_{2} F\right|_{(0, y)}(0, u)=-\operatorname{div}(\nabla u)($ recall $y=y(\Omega))$. By the standard theory of Dirichlet boundary value problem on compact Riemannian manifolds ([1], Theorem 4.8, p. 105 and [2], Theorem 7.32, p. 259),

$$
\left.D_{2} F\right|_{(0, y)}: H^{2}(\Omega) \cap H_{0}^{1}(\Omega) \longrightarrow L^{2}(\Omega)
$$

is an isomorphism. By (2.2), $F\left(t, y^{t}\right)=0 \forall t$. Proposition 2.1 now follows by the implicit function theorem.

\section{DEFINITION}

$\dot{y}(\Omega, V):=\left.\left(\frac{\mathrm{d}}{\mathrm{d} t} t^{t}\right)\right|_{t=0} \in H_{0}^{1}(\Omega)$ is called the (strong) material derivative of $y$ in the direction of $V$.

Consider $\Omega^{\prime} \subset \subset \Omega$. 


\section{PROPOSITION 2.2.}

The map $\left.t \longmapsto y_{t}\right|_{\Omega^{\prime}}$ is a $\mathscr{C}^{1}$-curve in $H^{1}\left(\Omega^{\prime}\right)$ from a neighbourhood of 0 in $\mathbb{R}$ and $\mathrm{d} /\left.\mathrm{d} t\right|_{t=0}\left(\left.y_{t}\right|_{\Omega^{\prime}}\right)=\left.\{\dot{y}(\Omega, V)-g(\nabla y, V)\}\right|_{\Omega^{\prime}}$.

Proof. There exists $\delta>0$ such that $\Omega^{\prime} \subset \Phi_{t}(\Omega) \forall|t|<\delta$. Then $\left.y_{t}\right|_{\Omega^{\prime}}=\left.y^{t} \circ \Phi_{-t}\right|_{\Omega^{\prime}} \forall|t|<$ $\delta$. Proposition 2.2 now follows from Proposition 2.1 and Proposition 2.38, p. 71 of [5]. $\square$

\section{DEFINITION}

$y^{\prime}(\Omega, V):=\dot{y}(\Omega, V)-g(\nabla y, V) \in H^{1}(\Omega)$ is called the shape derivative of $y$ in the direction of $V$.

Consider the domain functional $J\left(\Omega_{t}\right)$ defined by $J\left(\Omega_{t}\right):=\int_{\Omega_{t}} y_{t} \mathrm{~d} V(t \in \mathbb{R})$.

\section{DEFINITION}

The Eulerian derivative $\mathrm{d} J(\Omega, V)$ of $J\left(\Omega_{t}\right)$ at $t=0$ is defined as

$$
\mathrm{d} J(\Omega, V):=\lim _{t \rightarrow 0} \frac{J\left(\Omega_{t}\right)-J(\Omega)}{t} .
$$

\section{PROPOSITION 2.3.}

The function $J\left(\Omega_{t}\right)$ is differentiable at $t=0$ and $\mathrm{d} J(\Omega, V)=\int_{\Omega} y^{\prime} \mathrm{d} V$.

Proof. Let $L_{V} \omega$ denote the Lie derivative of $\omega$ with respect to $V$, and $i_{V} \omega$ denote the interior multiplication of $\omega$ with respect to $V$. Then

$$
\left.\frac{\mathrm{d}}{\mathrm{d} t}\left(\Phi_{t}^{*} \omega\right)\right|_{t=0}=: L_{V} \omega=\left(d i_{V}+i_{V} d\right) \omega=\mathrm{d}\left(i_{V} \omega\right)=\operatorname{div}(V) \omega .
$$

Hence, by Propositions 2.1 and 2.2 we get

$$
\begin{aligned}
\mathrm{d} J(\Omega, V) & =\lim _{t \rightarrow 0} \int_{\Omega}\left\{\frac{y^{t} \Phi_{t}^{*} \omega-y \omega}{t}\right\}=\int_{\Omega}\left(\frac{\mathrm{d}}{\mathrm{d} t}\left\{y^{t} \Phi_{t}^{*} \omega\right\}\right)_{\left.\right|_{t=0}} \\
& =\int_{\Omega}\{\dot{y}+y \operatorname{div}(V)\} \mathrm{d} V=\int_{\Omega}\left\{y^{\prime}+g(\nabla y, V)+y \operatorname{div}(V)\right\} \mathrm{d} V \\
& =\int_{\Omega} y^{\prime} \mathrm{d} V+\int_{\Omega} \mathrm{d}\left(y i_{V} \omega\right)=\int_{\Omega} y^{\prime} \mathrm{d} V .
\end{aligned}
$$

\section{PROPOSITION 2.4.}

The shape derivative $y^{\prime}=y^{\prime}(\Omega, V)$ is the weak solution of the Dirichlet boundary value problem

$$
\left.\begin{array}{rl}
\Delta v & =0 \quad \text { on } \Omega, \\
\left.v\right|_{\partial \Omega} & =-\frac{\partial y}{\partial n} g(V, n)
\end{array}\right\}
$$

in the space $H^{1}(\Omega)$. (Here, $n$ is the outward unit normal field on $\partial \Omega$ ). 
Proof. Consider $\psi \in \mathscr{C}_{0}^{\infty}(\Omega)$ having support in a domain $\Omega^{\prime} \subset \subset \Omega$. There exists $\delta>0$ such that $\Omega^{\prime} \subset \Omega_{t} \forall|t|<\delta$. By problem (2.1),

$$
\int_{\Omega^{\prime}} g\left(\nabla y_{t}, \nabla \psi\right) \mathrm{d} V=-\int_{\Omega^{\prime}} f \psi \mathrm{d} V \text { for }|t|<\delta .
$$

By Proposition 2.2, differentiation of LHS of eq. (4) with respect to $t$ at $t=0$ can be carried out under the integral sign. So we get

$$
\int_{\Omega^{\prime}} g\left(\nabla y^{\prime}, \nabla \psi\right) \mathrm{d} V=0 .
$$

Thus $y^{\prime}$ satisfies $\Delta y^{\prime}=0$ weakly on $\Omega$.

Now $\dot{y}, y \in H^{2}(\Omega) \cap H_{0}^{1}(\Omega)$, and $y^{\prime}=\dot{y}-g(\nabla y, V) \in H^{1}(\Omega)$. So by Proposition 2.39, p. 88 of [2], we get

$$
\left.y^{\prime}\right|_{\partial \Omega}=\left.\dot{y}\right|_{\partial \Omega}-\left.g(\nabla y, V)\right|_{\partial \Omega} \quad \text { and }\left.\quad \dot{y}\right|_{\partial \Omega}=0 .
$$

Also, $y \in \mathscr{C}^{\infty}(\bar{\Omega})$ and $y=0$ on $\partial \Omega$ by (2.1). So, $\left.g(\nabla y, V)\right|_{\partial \Omega}=\frac{\partial y}{\partial n} g(V, n)$. Thus, $\left.y^{\prime}\right|_{\partial \Omega}=$ $-\frac{\partial y}{\partial n} g(V, n)$.

\section{Shape calculus for the eigenvalue problem}

Let $(M, g), V, \Phi_{t}, \Omega, \Omega_{t}, \gamma_{t}, A_{t}$ be as in $\S 2$. Consider problem (1.2) posed in $\Omega_{t}$ :

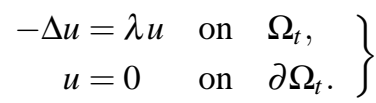

Let $\lambda_{1}(t):=\lambda_{1}\left(\Omega_{t}\right)$ and $y_{1}(t):=y_{1}\left(\Omega_{t}\right)$ be as in $\S 1$. We denote $y_{1}(\Omega)$ by $y_{1}$ and $\lambda_{1}(\Omega)$ by $\lambda_{1}$ throughout this section.

Denote $\left.y_{1}(t) \circ \Phi_{t}\right|_{\Omega}$ by $y_{1}^{t}(t \in \mathbb{R})$.

\section{PROPOSITION 3.1.}

The map $t \longmapsto\left(\lambda_{1}(t), y_{1}^{t}\right)$ is a $\mathscr{C}^{1}$-curve in $\mathbb{R} \times H^{2}(\Omega) \cap H_{0}^{1}(\Omega)$ from a neighbourhood of 0 in $\mathbb{R}$.

Proof. By problem (3.1), for each $t \in \mathbb{R}, y_{1}(t)$ satisfies the equation

$$
\int_{\Omega_{t}} g\left(\nabla y_{1}(t), \nabla \psi\right) \mathrm{d} V=\int_{\Omega_{t}} \lambda_{1}(t) y_{1}(t) \psi \mathrm{d} V \quad \forall \psi \in H_{0}^{1}\left(\Omega_{t}\right) .
$$

As in the proof of Proposition 2.1, eq. (5) can be re-written as

$$
-\int_{\Omega} \operatorname{div}\left(A_{t} \nabla y_{1}^{t}\right) \psi \mathrm{d} V=\int_{\Omega} \lambda_{1}(t) y_{1}^{t} \gamma_{t} \psi \mathrm{d} V \quad \forall \psi \in H_{0}^{1}(\Omega) .
$$

Therefore, $t \longmapsto\left(\lambda_{1}(t), y_{1}^{t}\right)$ satisfies

$$
\left.\begin{array}{rl}
\operatorname{div}\left(A_{t} \nabla y_{1}^{t}\right)+\lambda_{1}(t) y_{1}^{t} \gamma_{t} & =0 \quad \text { on } \Omega, \\
\int_{\Omega}\left(y_{1}^{t}\right)^{2} \gamma_{t} \mathrm{~d} V & =1 .
\end{array}\right\}
$$

Let $X:=\mathbb{R} \times H^{2}(\Omega) \cap H_{0}^{1}(\Omega)$. Define $F: \mathbb{R} \times X \longrightarrow L^{2}(\Omega) \times \mathbb{R}$ by $F(t, \mu, u)=$ $\left(\operatorname{div}\left(A_{t} \nabla u\right)+\mu u \gamma_{t}, \int_{\Omega} u^{2} \gamma_{t} \mathrm{~d} V-1\right)$. Then $F$ is a $\mathscr{C}^{1}$-map. Further $\left.D_{2} F\right|_{\left(0, \lambda_{1}, y_{1}\right)}$ $(0, \mu, u)=\left(\Delta u+\lambda_{1} u+\mu y_{1}, 2 \int_{\Omega} y_{1} u \mathrm{~d} V\right)$. 
Claim. $\left.D_{2} F\right|_{\left(0, \lambda_{1}, y_{1}\right)}: \mathbb{R} \times H^{2}(\Omega) \cap H_{0}^{1}(\Omega) \longrightarrow L^{2}(\Omega) \times \mathbb{R}$ is an isomorphism.

Let $(v, b) \in L^{2}(\Omega) \times \mathbb{R}$ be arbitrary. Consider the following problem:

$$
\left.\begin{array}{rl}
\Delta u+\lambda_{1} u+\mu y_{1} & =v \quad \text { on } \Omega, \\
2 \int_{\Omega} y_{1} u \mathrm{~d} V & =b .
\end{array}\right\}
$$

Now by Fredholm alternative, $\Delta u+\lambda_{1} u=v-\mu y_{1}$ has a solution in $H^{2}(\Omega) \cap H_{0}^{1}(\Omega)$ if and only if $v-\mu y_{1} \perp y_{1}$ in $L^{2}(\Omega)$. So, for $\mu_{0}:=\int_{\Omega} v y_{1} \mathrm{~d} V$ there exists $u_{1} \in H^{2}(\Omega) \cap H_{0}^{1}(\Omega)$ such that $\Delta u_{1}+\lambda_{1} u_{1}+\mu_{0} y_{1}=v$. Moreover, the solutions of $\Delta u+\lambda_{1} u+\mu_{0} y_{1}=v$ are of the form $u=u_{1}+a y_{1}, a \in \mathbb{R}$. Given $b \in \mathbb{R}$ there exists a unique $a_{0}:=b / 2-\int_{\Omega} y_{1} u_{1} \mathrm{~d} V \in \mathbb{R}$ such that $2 \int_{\Omega} y_{1} u \mathrm{~d} V=b$. Put $u_{0}=u_{1}+a_{0} y_{1}$. Thus for $(v, b) \in L^{2}(\Omega) \times \mathbb{R}$ there exists a unique $\left(\mu_{0}, u_{0}\right) \in \mathbb{R} \times H^{2}(\Omega) \cap H_{0}^{1}(\Omega)$ such that $\left.D_{2} F\right|_{\left(0, \lambda_{1}, y_{1}\right)}\left(0, \mu_{0}, u_{0}\right)=(v, b)$. This proves the claim.

By (3.2), $F\left(t, \lambda_{1}(t), y_{1}^{t}\right)=0 \forall t$. Proposition 3.1 now follows by the implicit function theorem.

\section{DEFINITION}

$\dot{y}_{1}(\Omega, V):=\left.\left((\mathrm{d} / \mathrm{d} t) y_{1}^{t}\right)\right|_{t=0} \in H_{0}^{1}(\Omega)$ is called the (strong) material derivative of $y_{1}$ in the direction of $V$.

Consider $\Omega^{\prime} \subset \subset \Omega$.

\section{PROPOSITION 3.2.}

The map $\left.t \longmapsto y_{1}(t)\right|_{\Omega^{\prime}}$ is a $\mathscr{C}^{1}$-curve in $H^{1}\left(\Omega^{\prime}\right)$ from a neighbourhood of 0 in $\mathbb{R}$ and $\left.\left((\mathrm{d} / \mathrm{d} t)\left[\left.y_{1}(t)\right|_{\Omega^{\prime}}\right]\right)\right|_{t=0}=\left.\left(\dot{y}_{1}-g\left(\nabla y_{1}, V\right)\right)\right|_{\Omega^{\prime}} \in H^{1}\left(\Omega^{\prime}\right)$. Further, $y_{1}^{\prime}$ satisfies $y_{1}^{\prime}=\dot{y}_{1}-$ $g\left(\nabla y_{1}, V\right)$ in $H^{1}(\Omega)$ and $\left.y_{1}^{\prime}\right|_{\partial \Omega}=-\frac{\partial y_{1}}{\partial n} g(V, n)$.

Proof. There exists $\delta>0$ such that $\Omega^{\prime} \subset \Phi_{t}(\Omega) \forall|t|<\delta$. The first part of Proposition 3.2 follows from Proposition 3.1 and Proposition 2.38, p. 71 of [5]. Now as $\dot{y}_{1} \in$ $H^{1}(\Omega)$ and $\nabla y_{1} \in \mathscr{C}^{\infty}(\bar{\Omega})$, we get $y_{1}^{\prime}=\dot{y}_{1}-g\left(\nabla y_{1}, V\right) \in H^{1}(\Omega)$. Hence, $\left.y_{1}^{\prime}\right|_{\partial \Omega}=\left.\dot{y}_{1}\right|_{\partial \Omega}-$ $\left.g\left(\nabla y_{1}, V\right)\right|_{\partial \Omega}=-\frac{\partial y_{1}}{\partial n} g(V, n)$.

\section{DEFINITION}

The shape derivative of $y_{1}$ in the direction of $V$ is the element $y_{1}^{\prime}=y_{1}^{\prime}(\Omega, V) \in H^{1}(\Omega)$ defined by $y_{1}^{\prime}=\dot{y}_{1}-g\left(\nabla y_{1}, V\right)$.

\section{PROPOSITION 3.3.}

The shape derivative $y_{1}^{\prime} \in H^{1}(\Omega)$ satisfies

$$
-\Delta y_{1}^{\prime}=\lambda_{1} y_{1}^{\prime}+\lambda_{1}^{\prime} y_{1} \quad \text { on } \Omega
$$

in the sense of distributions. 
Proof. Let $\psi \in \mathscr{C}_{0}^{\infty}(\Omega)$. Let $\Omega^{\prime} \subset \subset \Omega$ be a domain such that supp $\psi \subset \Omega^{\prime}$. As $y_{1}(t)$ is a solution of problem (1.2) posed in $\Omega_{t}$, for $t$ sufficiently small we get

$$
\int_{\Omega^{\prime}} g\left(\nabla y_{1}(t), \nabla \psi\right) \mathrm{d} V=\int_{\Omega^{\prime}} \lambda_{1}(t) y_{1}(t) \psi \mathrm{d} V .
$$

By Propositions 3.1 and 3.2, we can differentiate with respect to $t$ under the integral sign in eq. (7). Thus we have

$$
\int_{\Omega^{\prime}} g\left(\nabla y_{1}^{\prime}, \nabla \psi\right) \mathrm{d} V=\int_{\Omega^{\prime}}\left(\lambda_{1} y_{1}^{\prime}+\lambda_{1}^{\prime} y_{1}\right) \psi \mathrm{d} V
$$

Hence,

$$
-\int_{\Omega} y_{1}^{\prime} \Delta \psi \mathrm{d} V=\int_{\Omega}\left(\lambda_{1} y_{1}^{\prime}+\lambda_{1}^{\prime} y_{1}\right) \psi \mathrm{d} V \quad \forall \psi \in \mathscr{C}_{0}^{\infty}(\Omega)
$$

PROPOSITION 3.4.

$$
y_{1}^{\prime} \in \mathscr{C}^{\infty}(\bar{\Omega}) .
$$

Proof. By Proposition 3.2, $y_{1}^{\prime}=\dot{y}_{1}-g\left(\nabla y_{1}, V\right)$ on $\Omega$. Hence it is enough to prove that $\dot{y}_{1} \in \mathscr{C}^{\infty}(\bar{\Omega})$. Consider $L:=\Delta+\lambda_{1}$, a linear elliptic operator of order 2 . Then $\dot{y}_{1} \in H_{0}^{1}(\Omega)$ satisfies $L\left(\dot{y}_{1}\right)=L\left(y_{1}^{\prime}+g\left(\nabla y_{1}, V\right)\right)=-\lambda_{1}^{\prime} y_{1}+L\left(g\left(\nabla y_{1}, V\right)\right)$, by Proposition 3.3. From Proposition 3.58, p. 87 of [1], it follows that $\dot{y}_{1} \in \mathscr{C}^{\infty}(\bar{\Omega})$.

PROPOSITION 3.5.

$$
\lambda_{1}^{\prime}=-\int_{\partial \Omega}\left(\frac{\partial y_{1}}{\partial n}\right)^{2} g(V, n) \mathrm{d} S
$$

Proof. We write $\lambda_{1}^{\prime}=\lambda_{1}^{\prime} \int_{\Omega} y_{1}^{2} \mathrm{~d} V$. By Proposition 3.3, $\lambda_{1}^{\prime}=\int_{\Omega}\left\{-\Delta y_{1}^{\prime}-\lambda_{1} y_{1}^{\prime}\right\} y_{1} \mathrm{~d} V$. Hence by problem (1.2) and Proposition 3.4, we get

$$
\begin{aligned}
\lambda_{1}^{\prime}=\int_{\Omega}\left\{-y_{1} \Delta y_{1}^{\prime}+y_{1}^{\prime} \Delta y_{1}\right\} \mathrm{d} V & =\int_{\partial \Omega}\left\{y_{1}^{\prime} \frac{\partial y_{1}}{\partial n}-y_{1} \frac{\partial y_{1}^{\prime}}{\partial n}\right\} \mathrm{d} S \\
& =\int_{\partial \Omega} y_{1}^{\prime} \frac{\partial y_{1}}{\partial n} \mathrm{~d} S .
\end{aligned}
$$

Now the result follows by Proposition 3.2.

\section{Proofs of Theorem 1 and Theorem 2 for $S^{n}$}

Proof of Theorem 1 for $S^{n}$. We continue with the notations of $\S 1$ such as $r_{0}, r_{1}, \mathscr{F}$, and $y(\Omega), J(\Omega)$ for $\Omega \in \mathscr{F}$ for $S^{n}$. For $|t|<\pi$, put $p:=(0, \ldots, 0,1)$ and $q(t)=$ $(0, \ldots, 0, \sin t, \cos t) \in S^{n}$. The Laplace-Beltrami operator $\Delta$ of $\left(S^{n},\langle\rangle,\right)$ is invariant under isometries of $S^{n}$. So we need to study the functional $J$ only on domains $\Omega(q(t)):=B\left(r_{1}\right) \backslash \overline{B\left(q(t), r_{0}\right)}, 0 \leq|t|<r_{1}-r_{0}$, where $B\left(r_{1}\right):=B\left(p, r_{1}\right)$.

We define $j:\left(r_{0}-r_{1}, r_{1}-r_{0}\right) \longrightarrow \mathbb{R}$ by $j(t)=J(\Omega(q(t)))$. 
Fix $t_{0}$ such that $0 \leq t_{0}<r_{1}-r_{0}$ and put $\Omega:=\Omega\left(q\left(t_{0}\right)\right)$ and $B_{0}:=B\left(q\left(t_{0}\right), r_{0}\right)$. Fix $r_{2}$ such that $r_{0}<r_{2}<r_{1}-t_{0}$ and consider a smooth function $\rho: S^{n} \longrightarrow \mathbb{R}$ satisfying $\rho=1$ on $\overline{B\left(q\left(t_{0}\right), r_{2}\right)}$ and $\rho=0$ on $\partial B\left(r_{1}\right)$. Let $V$ denote the vector field on $S^{n}$ defined by $V(x)=\rho(x)\left(0, \ldots, 0, x_{n+1},-x_{n}\right) \forall x=\left(x_{1}, \ldots, x_{n+1}\right) \in S^{n}$. Let $\left\{\Phi_{t}\right\}_{t \in \mathbb{R}}$ be the oneparameter family of diffeomorphisms of $S^{n}$ associated with $V$. Then for $t$ sufficiently close to $0, J\left(\Phi_{t}(\Omega)\right)=j\left(t_{0}+t\right)$. Note that $J\left(\Phi_{t}(\Omega)\right)=\int_{\Omega_{t}} y_{t} \mathrm{~d} V$, hence by Proposition $2.3, j$ is differentiable at $t_{0}$.

Note that $j$ is an even function which is differentiable at 0 . Hence $j^{\prime}(0)=0$.

Now onwards we fix $t_{0}$ such that $0<t_{0}<r_{1}-r_{0}$ and consider $\Omega:=\Omega\left(q\left(t_{0}\right)\right)$ and $B_{0}:=B\left(q\left(t_{0}\right), r_{0}\right)$. Let $n$ denote the outward unit normal of $\Omega$ on $\partial \Omega$. For $x \in \partial B_{0}$, put $a=d(p, x)$ and $\alpha=$ the angle at $p$ of the spherical triangle $T:=\left[p, q\left(t_{0}\right), x\right]$ with vertices $p, q\left(t_{0}\right)$ and $x$. Then $n(x)=\left(q\left(t_{0}\right)-\cos r_{0} x\right) / \sin r_{0}$ and $\langle V, n\rangle(x)=\left(\cos a \sin t_{0}-\right.$ $\left.\sin a \cos t_{0} \cos \alpha\right) / \sin r_{0}$. Hence, by eq. (19) on p. 30 of [6], we get

$$
\langle V, n\rangle(x)=\cos \beta(x),
$$

where $\beta(x)$ denotes the angle at $q\left(t_{0}\right)$ of the spherical triangle $T$ defined above.

By Proposition 2.3, $j^{\prime}\left(t_{0}\right)=\int_{\Omega} y^{\prime} \mathrm{d} V$. Hence by Proposition 2.4 and problem (1.1),

$$
\begin{aligned}
\int_{\Omega} y^{\prime} \mathrm{d} V=-\int_{\Omega}\left\{y^{\prime} \Delta y-y \Delta y^{\prime}\right\} \mathrm{d} V & =-\int_{\partial \Omega}\left\{y^{\prime} \frac{\partial y}{\partial n}-y \frac{\partial y^{\prime}}{\partial n}\right\} \mathrm{d} S \\
& =-\int_{\partial \Omega} y^{\prime} \frac{\partial y}{\partial n} \mathrm{~d} S .
\end{aligned}
$$

Again by Proposition 2.4 and eq. (8) above, we get

$$
j^{\prime}\left(t_{0}\right)=\int_{x \in \partial B_{0}}\left(\frac{\partial y}{\partial n}(x)\right)^{2} \cos \beta(x) \mathrm{d} S .
$$

Let $H$ denote the hyperplane in $\mathbb{R}^{n+1}$ through $(0, \ldots, 0)$ having $q^{\prime}\left(t_{0}\right)$ as a normal vector. Let $r_{H}$ denote the reflection of $S^{n}$ about $H$. Put $\mathscr{O}=\left\{x \in \Omega \mid\left\langle x, q^{\prime}\left(t_{0}\right)\right\rangle>0\right\}$. Then $r_{H}(\mathscr{O}) \subset B\left(r_{1}\right)$ and $r_{H}\left(\overline{B_{0}}\right)=\overline{B_{0}}$. For $x \in \partial B_{0} \cap \partial \mathscr{O}$, let $x^{\prime}$ denote $r_{H}(x)$. Note that for all $x \in \partial B_{0} \cap \partial \mathscr{O}, \cos \beta(x)<0$ and $\cos \beta\left(x^{\prime}\right)=-\cos \beta(x)$. Thus eq. (9) can be re-written as

$$
j^{\prime}\left(t_{0}\right)=\int_{x \in \partial B_{0} \cap \partial \mathscr{O}}\left\{\left(\frac{\partial y}{\partial n}(x)\right)^{2}-\left(\frac{\partial y}{\partial n}\left(x^{\prime}\right)\right)^{2}\right\} \cos \beta(x) \mathrm{d} S .
$$

The Laplace-Beltrami operator $\Delta$ of $S^{n}$ is uniformly elliptic on $S^{n}$ and hence the maximum principle ([4], Theorem 5, p. 61) and the Hopf maximum principle ([4], Theorem 7, p. 65) are applicable on $\bar{\Omega}$. Hence, by arguments analogous to [3] at this stage, we get

$$
\left|\frac{\partial y}{\partial n}(x)\right|<\left|\frac{\partial y}{\partial n}\left(x^{\prime}\right)\right| \quad \forall x \in \partial B_{0} \cap \partial \mathscr{O}
$$

Thus from eq. (10), $j^{\prime}\left(t_{0}\right)>0$. This completes the proof of Theorem 1 for $S^{n}$.

Proof of Theorem 2 for $S^{n}$. We continue with the notations of $\S 1$ such as $\lambda_{1}(\Omega), y_{1}(\Omega)$ and $J_{1}(\Omega)$ for $\Omega \in \mathscr{F}$. Let $p, q(t)$ be as in the proof of Theorem 1. Define $j_{1}:\left(r_{0}-\right.$ 
$\left.r_{1}, r_{1}-r_{0}\right) \longrightarrow \mathbb{R}$ by $j_{1}(t)=J_{1}(\Omega(q(t)))$. As in the proof of Theorem 1, fix $t_{0}$ such that $0 \leq t_{0}<r_{1}-r_{0}$ and put $\Omega:=\Omega\left(q\left(t_{0}\right)\right)$ and $B_{0}:=B\left(q\left(t_{0}\right), r_{0}\right)$. Then for $t$ sufficiently close to 0 we have $j_{1}\left(t_{0}+t\right)=J_{1}\left(\Phi_{t}(\Omega)\right)=\lambda_{1}\left(\Phi_{t}(\Omega)\right)$. By Proposition 3.1, $j_{1}$ is differentiable at $t=t_{0}$ and $j_{1}^{\prime}\left(t_{0}\right)=\lambda_{1}^{\prime}(\Omega)$. As $\lambda_{1}\left(\Phi_{t}(\Omega)\right)=\lambda_{1}\left(\Phi_{-t}(\Omega)\right), j_{1}$ is an even function which is differentiable at 0 . Thus $j_{1}^{\prime}(0)=0$.

Now onwards we fix $t_{0}$ such that $0<t_{0}<r_{1}-r_{0}$ and put $\Omega:=\Omega\left(q\left(t_{0}\right)\right)$ and $B_{0}:=$ $B\left(q\left(t_{0}\right), r_{0}\right)$. Then by Proposition 3.5 and eq. (8), we get

$$
j_{1}^{\prime}\left(t_{0}\right)=\lambda_{1}^{\prime}(\Omega)=-\int_{\partial \Omega}\left(\frac{\partial y_{1}}{\partial n}\right)^{2}\langle V, n\rangle \mathrm{d} S=-\int_{\partial B_{0}}\left(\frac{\partial y_{1}}{\partial n}\right)^{2} \cos \beta(x) \mathrm{d} S .
$$

As in the proof of Theorem 1, eq. (11) can be re-written as

$$
j_{1}^{\prime}\left(t_{0}\right)=-\int_{x \in \partial B_{0} \cap \partial \mathscr{O}}\left\{\left(\frac{\partial y_{1}}{\partial n}(x)\right)^{2}-\left(\frac{\partial y_{1}}{\partial n}\left(x^{\prime}\right)\right)^{2}\right\} \cos \beta(x) \mathrm{d} S .
$$

The Laplace-Beltrami operator $\Delta$ of $S^{n}$ is uniformly elliptic on $S^{n}$. So, the Hopf maximum principle ([4], Theorem 7, p. 65) and the generalised maximum principle ([4], Theorem 10, p. 73) are applicable on $\bar{\Omega}$. Hence, by arguments analogous to [3] we get

$$
\left|\frac{\partial y_{1}}{\partial n}(x)\right|<\left|\frac{\partial y_{1}}{\partial n}\left(x^{\prime}\right)\right| \quad \forall x \in \partial B_{0} \cap \partial \mathscr{O} .
$$

It follows from eq. (12) that $j_{1}^{\prime}\left(t_{0}\right)<0$. The proof of Theorem 2 is now complete for $S^{n}$.

Remark on proofs of Theorem 1 and Theorem 2 for $\mathbb{H}^{n}$. For $t \in \mathbb{R}$, define $q(t)=(0, \ldots, 0, \sinh t, \cosh t) \in$ $\mathbb{H}^{n}$. Put $p:=q(0)$ and $q:=q\left(t_{0}\right)\left(t_{0}>0\right)$. Define the vector field $V$ on $\mathbb{H}^{n}$ by $V(x)=\rho(x)\left(0, \ldots, 0, x_{n+1}, x_{n}\right) \forall x=\left(x_{1}, \ldots, x_{n+1}\right) \in \mathbb{H}^{n}$, where $\rho: \mathbb{H}^{n} \longrightarrow \mathbb{R}$ is as in the proof of Theorem 1 for $S^{n}$.

Let $n$ denote the inward unit normal of $B\left(q, r_{0}\right)$ on $\partial B\left(q, r_{0}\right)$. Then,

$$
n(x)=\left(q-\cosh r_{0} x\right) / \sinh r_{0}
$$

and

$$
\langle V, n\rangle(x)=\left(x_{n+1} \sinh t_{0}-x_{n} \cosh t_{0}\right) / \sinh r_{0}=\cos \beta(x),
$$

where $\beta(x)$ denotes the angle at $q$ of the hyperbolic triangle $[p, q, x]$ with vertices $p, q$ and $x$.

Now Theorems 1 and 2 for the hyperbolic case can be proved using shape calculus of $\S \S 2$ and 3 as in the case of sphere.

\section{Acknowledgement}

The work of the first author is supported by research scholarship from the National Board for Higher Mathematics NBHM/RAwards.2001/643(1). 


\section{References}

[1] Aubin T, Nonlinear analysis on manifolds - Monge-Ampere equations (Springer-Verlag) (1982)

[2] Folland G, Introduction to partial differential equations (New Delhi: Prentice-Hall of India Private Limited) (2001)

[3] Kesavan S, On two functionals connected to the Laplacian in a class of doubly connected domains, Proc. R. Soc. Edinburgh 133A (2003) 617-624

[4] Protter $M$ and Weinberger H, Maximum principles in differential equations (New Jersey: Prentice-Hall Inc.) (1967)

[5] Sokolowski J and Zolesio J-P, Introduction to shape optimization - shape sensitivity analysis (Springer-Verlag) (1992)

[6] Todhunter I, Spherical Trigonometry (London: Macmillan and Co. Ltd) (1949) 